\title{
Endothelial protective effect of rapamycin against simulated ischemia injury through up-regulation of autophagy and inhibition of endoplasmic reticulum stress
}

Bożena Gabryel

Department of Pharmacology, School of Medicine, Medical University of Silesia, Katowice, Poland

Submitted: 21 October 2019

Accepted: 16 February 2020

Arch Med Sci Civil Dis 2020; 5: e14-e21

DOI: https://doi.org/10.5114/amscd.2020.94101

Copyright (c) 2020 Termedia \& Banach

\section{Abstract}

Introduction: Rapamycin has been shown to have cytoprotective properties in some experimental models of ischemia. However, the precise molecular mechanisms underlying the positive effect of rapamycin on endothelial cells in ischemic injury remain unknown. It is very important because endothelial cells are firstly exposed to ischemia and play an important role in ischemic organ damage. Autophagy and endoplasmic reticulum stress are suggested to be implicated in hypoxic/ischemic injury of endothelial cells. This study aims to explore whether the endothelial protective effect of rapamycin is associated with exacerbation of autophagy and attenuation of endoplasmic reticulum stress.

Material and methods: The protective effects of rapamycin against oxygen and glucose deprivation (OGD)-induced cell injury were explored in human vascular endothelial cells (HUVECS). Cell viability was measured by MTT assay. The protein levels of Beclin 1, p62, p-mTOR, p-S6K, p-4EBP, GRP78, p-PERK and p-IRE1 were analyzed using immunoblotting.

Results: Rapamycin in the simulated ischemia model increased the cell viability, indicating its cytoprotective effect $(p<0.05)$. Experiments with 3-methyladenine as an inhibitor of autophagy and thapsigargin as an inducer of endoplasmic reticulum stress support that rapamycin exerts endothelial protective effects against OGD-induced damage via autophagy - endoplasmic reticulum stress pathway.

Conclusions: This study demonstrated that rapamycin protects ischemic HUVECs via down-regulation of the mTOR pathway, enhancement of autophagy and inhibition of endoplasmic reticulum stress.

Key words: rapamycin, autophagy, ER stress, endothelial cells, ischemia.

\section{Introduction}

Autophagy is a catabolic process that is responsible for the elimination of aggregated proteins and damaged organelles [1]. In the initial step of autophagy the cytoplasmic components are sequestered in double-membrane vesicles, referred to as autophagosomes. Autophagosomes fuse with acidified endosomal and/or lysosomal vesicles to generate autolysosomes, where the luminal content is degraded and recycled [2]. The essential role of autophagy in most cells is the basal turnover of cellular

\author{
Corresponding author: \\ Bożena Gabryel PhD \\ Department \\ of Pharmacology \\ School of Medicine \\ Medical University \\ of Silesia \\ 18 Medyków St \\ 40-752 Katowice, Poland \\ Phone: +4832208 8525 \\ E-mail: bgabryel@interia.pl
}


components and maintenance of the function [1]. Autophagy principally is an important adaptive mechanism under different stress conditions such as exposure to hypoxia, ischemia or starvation [3]. Under ischemic/hypoxic stress induction of autophagy is considered as a process that promotes cell survival and counteracts apoptosis [4]. However, excessive autophagy may destroy essential cellular compounds and lead to cell death [2].

Beclin 1 and p62/SQSTM1 (p62) are the main autophagy markers. Beclin 1, a $\mathrm{BH} 3$-only domain protein, forms a complex with class III phosphoinositide 3-kinase (PI3K) responsible for autophagic vesicle nucleation and autophagy induction [5]. The p62 binds directly to microtubule-associated protein 1 light chain 3 (LC3) protein, is incorporated into the autophagosomes and degraded in autolysosomes. A measure of p62 expression is commonly used as a marker to study autophagic flux [6].

Accumulating evidence suggests that ischemic organ damage is linked to autophagy. In vivo studies performed in experimental models of ischemia show the increased expression of Beclin 1 and LC3-II [7-9]. However, it is still controversial whether autophagy is beneficial or fatal to ischemic tissue. Furthermore, the impact of autophagy induced by ischemia on endothelial cells (ECs) is unknown. ECs play a key role in regulation of blood flow, suppression of vascular inflammation and thrombosis as well as in release of trophic factors. Injury of ECs leads to platelet activation, increased reactive oxygen species production, inflammation in blood vessels and progression of ischemic tissue damage [10]. For this reason, the identification of the molecular mechanism of autophagy in ECs constitutes a potential target for the development of possible strategies to minimize ischemic injury.

Ischemia also contributes to the aggregation of misfolded or unfolded proteins within the endoplasmic reticulum (ER), which results in ER stress and unfolded protein response (UPR) [11]. There are three major ER-resident proteins involved in UPR, namely protein-kinase-RNA like ER kinase (PERK), inositol-requiring enzyme 1 (IRE1) and activating transcription factor (ATF6) [12]. Physiologically, their activity is suppressed by binding of the intraluminal chaperone glucose regulated protein 78 (GRP78). In response to ER stress, GRP78 is released and binds to the unfolded proteins, which leads to the activation of PERK, IRE1 and ATF6 [12]. Furthermore, there is evidence that ER stress is linked to autophagy [13, 14].

Numerous studies have demonstrated that $\mathrm{PI3K} / \mathrm{Akt} / \mathrm{mTOR}$ axis is involved in ischemic injury in various pathophysiological conditions [7, $15,16]$. mTOR has been reported to play a sig- nificant role in regulation of metabolism, gene transcription, protein synthesis and degradation, autophagy, apoptosis and necroptosis [17]. After activation, mTOR phosphorylates its downstream target proteins: ribosomal S6 kinase (S6K) and eukaryote initiation factor $4 \mathrm{E}$ binding protein 1 (4EBP1) [17]. It was shown that mTOR inhibition is associated with the up-regulation of autophagy and protective effects in experimental ischemia and reperfusion [7]. Rapamycin, an immunosuppressive agent and mTOR inhibitor, exerts cytoprotective effects in both cell and animal models of ischemia $[18,19]$. The available data suggest that rapamycin activates protective autophagy and inhibits ER stress in ischemic neurons, hepatocytes and nephrons [20-22]. However, whether up-regulation of autophagy and attenuation of ER stress by rapamycin has a protective effect on ischemic ECs remains to be elucidated.

In this paper the ability of rapamycin to induce autophagy and attenuate ER stress in human umbilical vein endothelial cells (HUVECs) following in vitro simulated ischemia injury was determined.

\section{Material and methods}

\section{Cell culture and treatment}

HUVECs (Lonza, USA) from passages 3 through 5 were cultured in EGM-2 medium (Lonza) supplemented with EGM-2 Bullet Kit (Lonza) under standard conditions. For simulated ischemia $\left(3 \% \mathrm{O}_{2}\right.$, no glucose), confluent cells in DMEM without glucose were transferred to a hypoxic chamber (Eppendorf Inc., USA) which was flushed with $92 \%$ $\mathrm{N}_{2}, 5 \% \mathrm{CO}_{2}$ and $3 \% \mathrm{O}_{2}$ at $37^{\circ} \mathrm{C}$. Cells were maintained under oxygen-glucose deprivation (OGD) for $6 \mathrm{~h}$ and treated with $100 \mathrm{nM}$ rapamycin (Rapa, Sigma-Aldrich, USA) without/with 3-methyladenine (3-MA, Sigma-Aldrich, $5 \mathrm{mM}$ ) or thapsigargin (TG, Sigma-Aldrich, $1 \mu \mathrm{M}$ ). The control groups (Normoxia) were placed in EGM-2 with EGM-2 Bullet Kit in the incubator with normal $\mathrm{O}_{2}$ content.

\section{Cell viability}

The cell viability was assessed by [3(4,5-dimethylthiazol-2-yl]-2,5 diphenyltetrazolium bromide (MTT, Sigma-Aldrich) assay using a final concentration of MTT of $0.25 \mathrm{mg} / \mathrm{ml}$ and $3 \mathrm{~h}$ incubation. The cells were lysed in $100 \mu \mathrm{DMSO}$ and optic density (OD) was measured on the microplate reader at $570 \mathrm{~nm}$.

\section{Western blot}

Cells were homogenized in RIPA lysis buffer. Twenty $\mu \mathrm{g}$ lysates per sample were separated on $10 \%$ SDS-PAGE gels, which were then transferred to PVDF membranes. After blocking with 5\% dry 


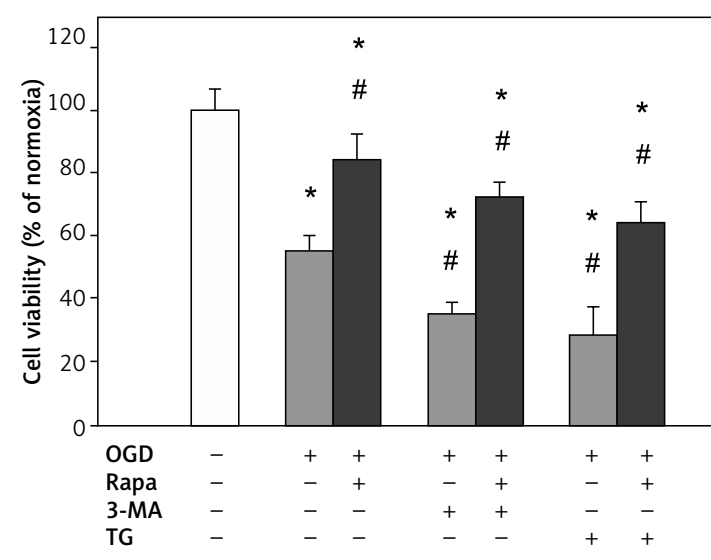

Figure 1. Rapamycin (Rapa) attenuates and 3-methyladenine (3-MA) and thapsigargin (TG) potentiate oxygen and glucose deprivation (OGD)-induced injury of HUVECs. Cell survival was measured by MTT assay

Results are mean $\pm S D(n=8),{ }^{*} p<0.05$ vs. Normoxia ${ }^{\#} p<0.05$ vs. OGD.

milk in TBST, the implied primary and secondary antibodies were added. Afterwards, the blots were developed using ECL reagents and OD was quantified with ImageJ software. The following primary antibodies purchased from Santa Cruz Biotechnology (Santa Cruz, USA) and dilutions were used: Beclin 1 (1/500), p62 (1/2000), p-mTOR (1/1000), mTOR (1/1000), p-S6K (1/500), S6K (1/500), p-4EBP1 (1/500), 4EBP1 (1/500), GRP78 (1/1000), p-PERK (1/1000), PERK (1/1000), p-IRE1 (1/1000), IRE1 (1/1000) and $\beta$-actin (1/1000).

\section{Statistical analysis}

Statistical significance was determined by oneway analysis of variance (ANOVA) followed by Bonferroni's multiple comparison test. A value of $p<0.05$ was accepted as statistical significance.

\section{Results}

\section{Rapamycin increases and 3-MA and TG decrease cell viability}

To determine the ability of rapamycin to protect OGD-injured HUVECs through autophagy activation and ER stress attenuation, the cells were treated with 3-MA and TG. 3-MA is a widely used inhibitor of autophagy acting by PI3K inhibition, and thus it suppresses autophagosome formation [23]. TG, a specific inhibitor of ER $\mathrm{Ca}^{2+}$-dependent ATPase, is ER stress inducer. Furthermore, it was shown that TG blocks autophagosome-lysosome fusion and inhibits autophagic flux [24]. The concentrations of 3-MA and TG $(5 \mathrm{mM}$ and $1 \mu \mathrm{M}$, respectively) were selected on the basis of studies carried out on HUVECs $[25,26]$.

The viability of HUVECs was significantly reduced by 6 h OGD treatment to 55\% ( $p<0.05$,
Figure 1). Compared with both Normoxia and OGD groups, 3-MA and TG substantially reduced HUVECS viability to $35 \%$ and $29 \%$, respectively $(p<0.05$, Figure 1$)$. In preliminary dose-response experiments, rapamycin (10-1000 nM) did not have any influence on cell survival for $6 \mathrm{~h}$ in normoxia (data not shown). In the present study, the cells were incubated with $100 \mathrm{nM}$ rapamycin. At this concentration, the autophagy induction and mTOR inhibition in ischemic HUVECs was observed (Figures 2 and 3). Moreover, the cell viability was significantly increased after simultaneous treatment with rapamycin with 3-MA or TG under OGD to $72 \%$ and $65 \%$ respectively, as compared with the OGD alone $(p<0.05)$.

\section{Rapamycin counters the inhibitory effects of 3-MA and TG on autophagy}

The expressions of Beclin 1 and p62 as important autophagy biomarkers were selected to assess the activation autophagy flux in HUVECs. As shown in Figure 2, a significant increase in Beclin 1 expression was observed following OGD $(p<0.05)$. Furthermore, 3-MA and TG treatment attenuated Beclin 1 expression, which was coincident with significant p62 up-regulation and reduced degradation $(p<0.05)$. Rapamycin significantly increased both the Beclin 1 level and p62 degradation (Figure 2, $p<0.05$ ). Furthermore, the drug was able to effectively counteract the blockade of autophagy flux by both 3-MA and TG treatment during OGD.

\section{OGD and rapamycin inhibit mTOR signaling pathway}

The mTOR pathway plays an essential role in pathophysiology of ischemic injury [27]. mTOR is a negative regulator of autophagy, e.g. activation of mTOR resulted in decreased autophagic flux [28]. The expressions of phosphorylated forms of S6K and 4EBP1 are indicators of mTOR activity [27].

As shown in Figure 3, the expressions of p-mTOR, p-S6K and p-4EBP1 were significantly decreased after OGD and rapamycin in comparison to normoxic cells $(p<0.05)$. In addition, total mTOR, S6K and 4-EBP1 levels were unchanged in all experimental groups. The results suggest that the mTOR pathway was effectively suppressed by rapamycin in this experimental model.

\section{Rapamycin attenuates ER stress in OGD-injured HUVECS}

To determine whether attenuation of ER stress is involved in the endothelial protective effect of rapamycin against OGD-induced injury, the expressions of ER stress-related proteins were determined. The analysis of Western blot data showed that the ex- 
A

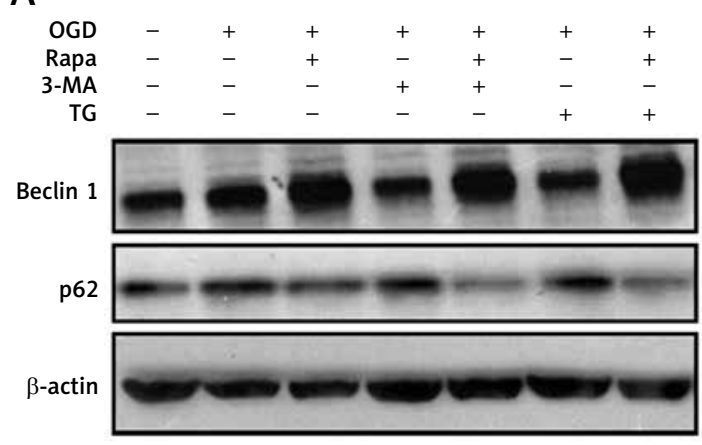

C

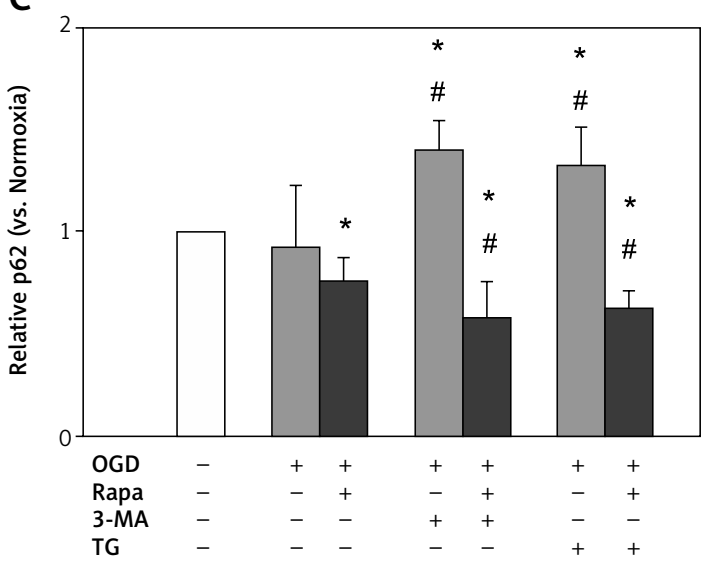

pression levels of GRP78, p-PERK and p-IRE1 were significantly increased in ischemic HUVECs revealing OGD-induced ER stress $(p<0.05$, Figure 4$)$. In the rapamycin-treated group, the expression levels of ER-stress related proteins were significantly decreased compared with OGD (Figure 4, $p<0.05$ ). It should be noted that the expressions of total PERK and IRE1 were not significantly changed. This demonstrated that rapamycin inhibited ER stress through down-regulating the phosphorylation of signaling proteins, e.g. p-PERK and p-IRE1.

\section{Discussion}

Autophagy is a catabolic process of degradation of cellular constituents [1]. Numerous reports have demonstrated that autophagy is impaired in ischemia/hypoxia and autophagosomes are accumulated within cells $[29,30]$. Moreover, it has been proposed that autophagy dysfunction in endothelium following ischemia might be involved in a breakdown of the endothelial barrier resulting in vascular hyperpermeability and induction of autophagy in ECs has a protective effect [31, 32]. However, the involvement of ER stress-mediated autophagy in endothelial dysfunction in response to ischemic injury remains unclear. On one hand, there is evidence that ER stress might contribute to activation of autophagy [9]. But on the other, it was shown that TG, an ER stress

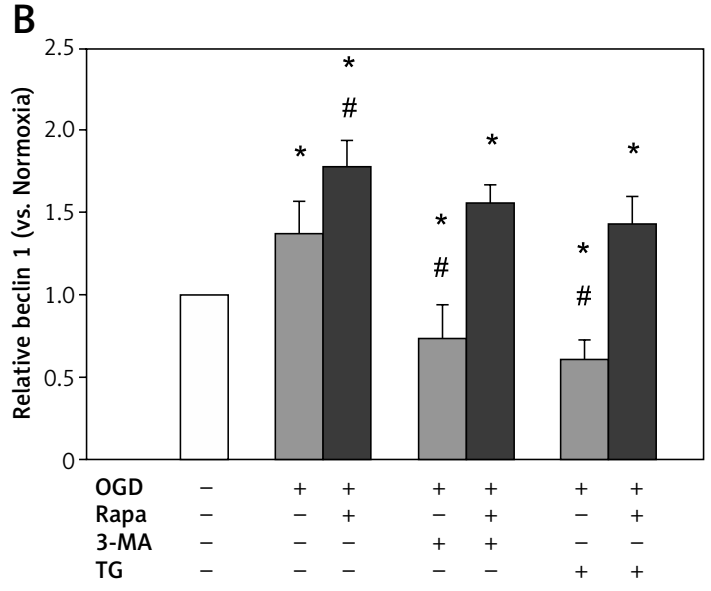

Figure 2. Rapamycin (Rapa) enhances autophagy in HUVECs exposed to oxygen and glucose deprivation (OGD) as detected by Western blot. A - Expression of Beclin 1 and $\mathrm{p} 62$ in HUVECs treated with $100 \mathrm{nM}$ Rapa or 5 mM 3-methyladenine (3-MA) and $1 \mu \mathrm{M}$ thapsigargin (TG) with or without Rapa. Blots are representative of three independent experiments. Densitometric analyses of Beclin-1 (B) and p62 (C) normalized to $\beta$-actin

Results are mean $\pm S D(n=3)$. ${ }^{*} p<0.05$ vs. Normoxia, ${ }^{\#} p<0.05$ vs. OGD.

inductor, is able to inhibit autophagy. TG-inhibited autophagy might result from block fusion of autophagosomes with lysosomes and failure of autophagic flux [24]. The presented results from MTT assay clearly indicate that both inhibition of autophagy by 3-MA and activation of ER stress by TG decreased viability of HUVECS under OGD (Figure 1). This is consistent with the observations by Xie et al. [33], who described the increased advanced glycation end product (AGE)-induced injury of HUVECs after 3-MA treatment. The authors suggested that early autophagy activation in ECS is involved in the protective mechanism and promotes cell survival [33].

It should be also noted that during normal growth conditions HUVECs expressed a relatively high basal level of autophagy as shown by marked Beclin 1 expression (Figure 2 A). The constitutive active basal autophagy in ECs might contribute to their resistance to ischemia by maintaining the sufficient level of intracellular ATP [8]. Instead, failure of basal autophagy causes the increase in ROS production that activates inflammatory processes and apoptosis [34, 35].

This study has shown that rapamycin increased cell viability following autophagy inhibition and ER stress induction in ischemic HUVECs (Figure 1). Moreover, inhibition of mTOR by rapamycin clearly raised Beclin 1 level and p62 degradation in 
A

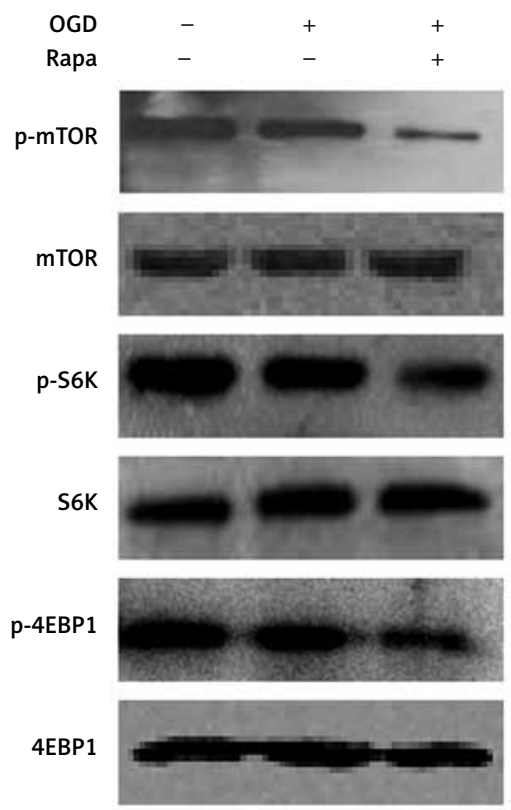

C

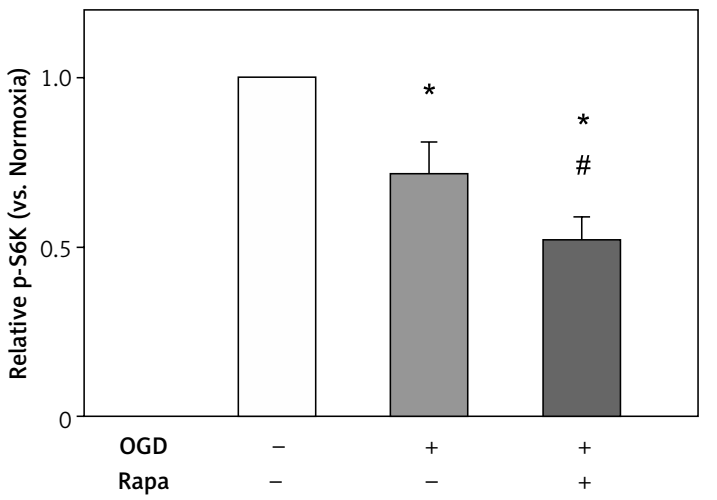

B

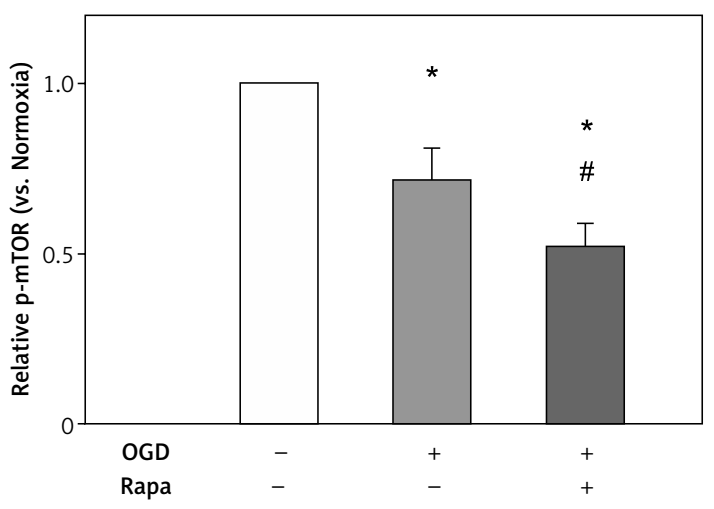

D

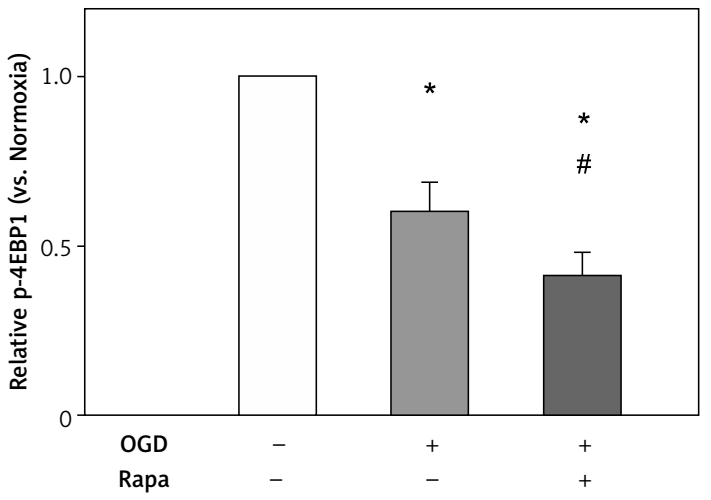

Figure 3. Rapamycin (Rapa) inhibits the mTOR pathway in HUVECs exposed to oxygen and glucose deprivation (OGD) as detected by Western blot. A - Expression of p-mTOR, mTOR, p-S6K, S6K, p-4EBP1 and 4EBP1 in HUVECs treated with $100 \mathrm{nM}$ Rapa. Blots are representative of three independent experiments. Densitometric analyses of p-mTOR (B), p-S6K (C) and p-4EBP1 (D) normalized to mTor, S6K and 4EBP1, respectively

Results are mean $\pm S D(n=3)$. ${ }^{*} p<0.05$ vs. Normoxia, ${ }^{*} p<0.05$ vs. OGD.

HUVECs treated with/without 3-MA or TG in OGD (Figure 2). The role of the mTOR pathway in ECs in various vascular disorders remains controversial. The inhibition of mTOR pathway using curcumin protects HUVECs from oxidative stress and activates of autophagy [30]. However, Xie et al. [36] showed that $\alpha$-lipoic acid through mTOR activation reduces OGD-induced endothelial injuries, thereby suggesting that down-regulation of the mTOR pathway may be unfavorable to ischemic ECs. It seems that these differences may result from distinct cell types and experimental ischemic models. In this study, rapamycin used as a mTOR inhibitor and autophagy agonist effectively protected ECs against ischemic injury.

mTOR is known as an universal regulator of autophagy. In experimental models of the cerebral stroke, it was shown that inhibition of mTOR by rapamycin activated autophagy in penumbra and had a neuroprotective effect $[7,18]$. This study also demonstrated that $\mathrm{mTOR}$ is a negative regulator of protective autophagy in OGD-injured HUVECs. Furthermore, the activation of autophagy by rapamycin in ischemic ECS was connected to the decreased phosphorylation levels of mTOR, S6K and 4EBP1 (Figure 3).

The molecular mechanism leading to the autophagy activation by rapamycin remains unknown. The available data indicate that rapamycin inactivates mTORC1, thereby enhancing autophagosome formation through ulc-51-like autophagy activating kinase 1 (ULK1) [37]. In addition, the autophagy induced by rapamycin in Beclin 1-dependent mechanism protects against neonatal ischemia [38, 39]. 
A

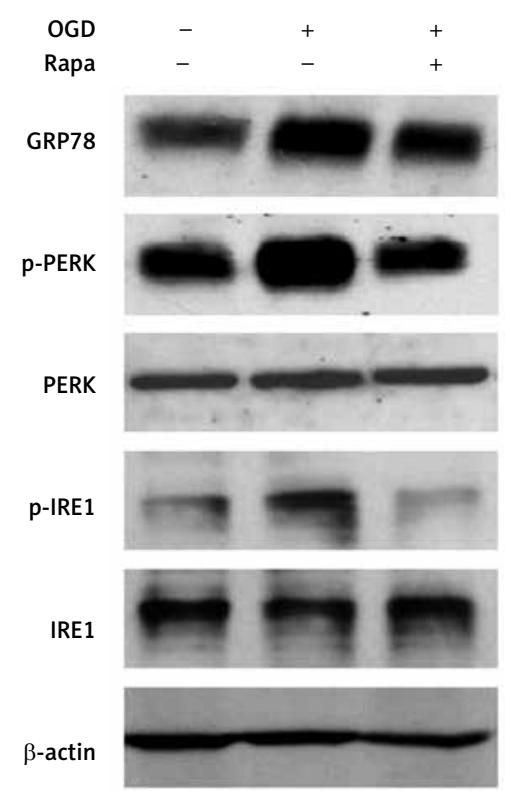

C

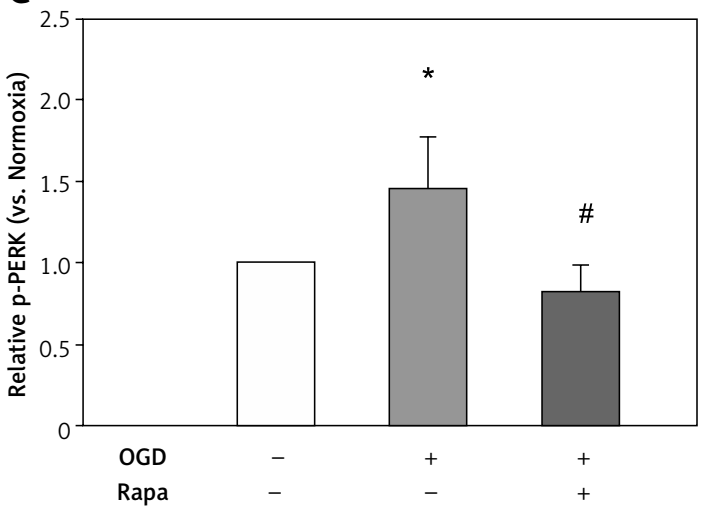

B

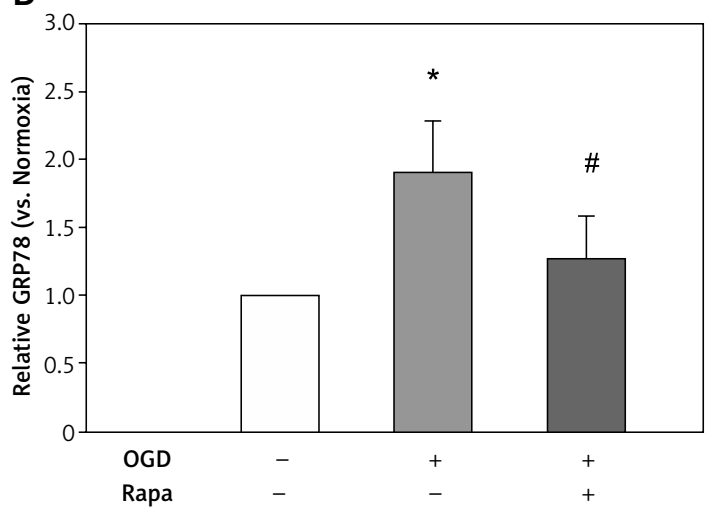

D

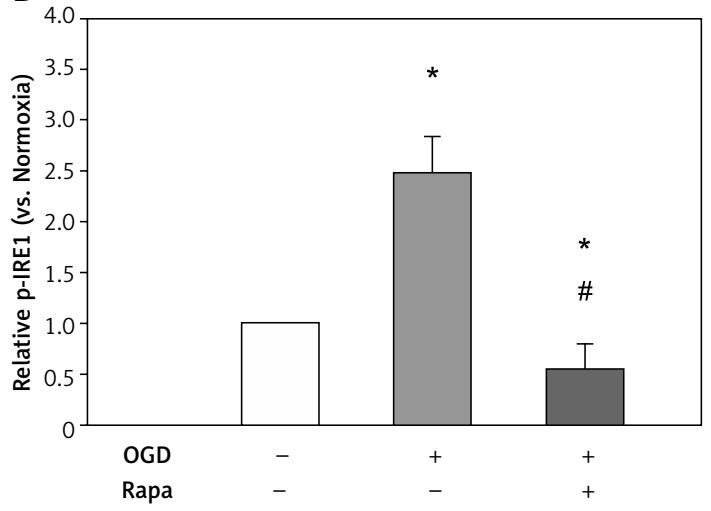

Figure 4. Rapamycin (Rapa) attenuates ER stress in HUVECs exposed to oxygen and glucose deprivation (OGD) as detected by Western blot. A - Expression of GRP78, p-PERK, PERK, p-IRE and IRE in HUVECs treated with $100 \mathrm{nM}$ Rapa. Blots are representative of three independent experiments. Densitometric analyses of GRP78 (B), p-PERK (C) and $p$-IRE1 (D) normalized to $\beta$-actin, PERK and IRE1, respectively

Results are mean $\pm S D(n=3) .{ }^{*} p<0.05$ vs. Normoxia, ${ }^{\#} p<0.05$ vs. OGD.

However, as mTOR modulates a number of cellular functions, the prevalence of events other than autophagy induction could be responsible for the endothelial protective effect of rapamycin.

It has been proposed that mild ER stress is an activator of protective autophagy in ischemic stroke. However, prolonged and severe ER stress leads to apoptosis and autophagy disturbances [40]. There is some evidence suggesting the crosstalk between ER stress and the mTOR signaling pathway [41]. In addition, the link between autophagy and ER stress at the level of molecules as Beclin 1 and ER-stress related proteins was suggested [9]. Yet, little is known about the molecular mechanisms underlying the interactions between autophagy and ER stress after rapamycin treatment. It was shown that rapamycin enhances cell survival under ER stress and protects against apoptosis [42-44]. Furthermore, significant suppression of ER-stress induced IRE1 pathway by rapamycin was described [42]. In an in vitro experimental model it was shown that inactivation of $\mathrm{mTOR}$ by rapamycin leads to an increase in cell survival under ER stress and activation of autophagy [45]. This study demonstrated that rapamycin inhibits the expression of ER-stress related proteins in OGD-injured HUVECs (Figure 4). At the same time, rapamycin induces protective autophagy and inhibits mTOR pathway (Figures 2 and 3).

In the present study, endothelial cell cultures were treated with rapamycin at a concentration of $100 \mathrm{nM}$ which does not have toxic effects and is commonly used in scientific works on cytoprotection. The concentration range of $10-100 \mathrm{nM}$ is 
used in most vitro studies on rapamycin in order to suppress mTORC1 and induce autophagy. The higher doses of rapamycin $(0.2-20 \mu \mathrm{M})$ targeting mTORC2 and having non-specific effects are far beyond that tolerated in humans [46].

In conclusion, the present study shows that OGD induces endothelial cell injury, activates autophagy, mTOR pathway and ER stress. Rapamycin has an apparent endothelial protective effect against ischemic damage through enhancement of autophagic flux together with inhibition of the mTOR pathway and ER stress-related proteins. The author is aware of the limitations of this study; the most important is that all experiments were performed only in in vitro conditions. Therefore, additional studies are needed to confirm these protective mechanisms of rapamycin towards ECS in experimental models of ischemia.

\section{Acknowledgments}

The work was partially supported by grant No. N N401 072139 from the Ministry of Sciences and Higher Education, Warsaw, Poland.

\section{Conflict of interest}

The authors declare no conflict of interest.

\section{References}

1. Kroemer G, Mariño G, Levine B. Autophagy and the integrated stress response. Mol Cell 2010; 40: 280-93.

2. Kang R, Zeh HJ, Lotze MT, Tang D. The Beclin 1 network regulates autophagy and apoptosis. Cell Death Differ 2011; 18: 571-80.

3. Sun Y, Zhu Y, Zhong X, Chen X, Wang J, Ying G. Crosstalk between autophagy and cerebral ischemia. Front $\mathrm{Neu}$ rosci 2019; 12: 1022.

4. Wang P, Shao BZ, Deng Z, Chen S, Yue Z, Miao CY. Autophagy in ischemic stroke. Prog Neurobiol 2018; 163 164: 98-117.

5. Sinha S, Levine B. The autophagy effector Beclin 1: a novel BH3-only protein. Oncogene 2008; 27 Suppl 1: S137-48.

6. Bjørkøy G, Lamark T, Pankiv S, Øvervatn A, Brech A, Johansen T. Monitoring autophagic degradation of p62/ SQSTM1. Methods Enzymol 2009; 452: 181-97.

7. Buckley KM, Hess DL, Sazonova IY, et al. Rapamycin up-regulation of autophagy reduces infarct size and improves outcomes in both permanent MCAL, and embolic MCAO, murine models of stroke. Exp Transl Stroke Med 2014; 6: 8

8. Matsui Y, Kyoi S, Takagi H, et al. Molecular mechanisms and physiological significance of autophagy during myocardial ischemia and reperfusion. Autophagy 2008; 4: 409-15.

9. Guan G, Yang L, Huang W, et al. Mechanism of interactions between endoplasmic reticulum stress and autophagy in hypoxia/reoxygenation-induced injury of $\mathrm{H} 9 \mathrm{c} 2$ cardiomyocytes. Mol Med Rep 2019; 20: 350-8.

10. Rajendran P, Rengarajan T, Thangavel J, et al. The vascular endothelium and human diseases. Int J Biol Sci 2013; 9: 1057-69.
11. Mei Y, Thompson MD, Cohen RA, Tong X. Endoplasmic reticulum stress and related pathological processes. J Pharmacol Biomed Anal 2013; 1: 1000107.

12. Sano R, Reed JC. ER stress-induced cell death mechanisms. Biochim Biophys Acta 2013; 1833: 3460-70.

13. Qin L, Wang Z, Tao L, Wang Y. ER stress negatively regulates AKT/TSC/mTOR pathway to enhance autophagy. Autophagy 2010; 6: 239-47.

14. Senft D, Ronai ZA. UPR, autophagy, and mitochondria crosstalk underlies the ER stress response. Trends Biochem Sci 2015; 40: 141-8.

15. Chi Y, Ma Q, Ding XQ, Qin X, Wang C, Zhang J. Research on protective mechanism of ibuprofen in myocardial ischemia-reperfusion injury in rats through the PI3K/ Akt/mTOR signaling pathway. Eur Rev Med Pharmacol Sci 2019; 23: 4465-73.

16. Wei Q, Zhao J, Zhou X, Yu L, Liu Z, Chang Y. Propofol can suppress renal ischemia-reperfusion injury through the activation of PI3K/AKT/mTOR signal pathway. Gene 2019; 708: 14-20.

17. Maiese K. Cutting through the complexities of mTOR for the treatment of stroke. Curr Neurovasc Res 2014; 11: 177-86.

18. Wu M, Zhang H, Kai J, et al. Rapamycin prevents cerebral stroke by modulating apoptosis and autophagy in penumbra in rats. Ann Clin TransI Neurol 2017; 5: 138-46.

19. Kocak Z, Temiz-Resitoglu M, Guden DS, et al. Modulation of oxidative/nitrosative stress and inflammatory response by rapamycin in target and distant organs in rats exposed to hindlimb ischemia/reperfusion: the role of mTOR. Can J Physiol Pharmacol 2019; 97: 1193-203.

20. Carloni S, Girelli S, Scopa C, Buonocore G, Longini M, Balduini W. Activation of autophagy and Akt/CREB signaling play an equivalent role in the neuroprotective effect of rapamycin in neonatal hypoxia-ischemia. Autophagy 2010; 6: 366-77.

21. Zhu J, Hua X, Li D, Zhang J, Xia Q. Rapamycin attenuates mouse liver ischemia and reperfusion injury by inhibiting endoplasmic reticulum stress. Transplant Proc 2015; 47: 1646-52.

22. Li X, Zhu G, Gou X, et al. Negative feedback loop of autophagy and endoplasmic reticulum stress in rapamycin protection against renal ischemia-reperfusion injury during initial reperfusion phase. FASEB J 2018: fj201800299R.

23. Vakifahmetoglu-Norberg H, Xia HG, Yuan J. Pharmacologic agents targeting autophagy. J Clin Invest 2015; 125: 5-13.

24. Ganley IG, Wong PM, Gammoh N, Jiang X. Distinct autophagosomal-lysosomal fusion mechanism revealed by thapsigargin-induced autophagy arrest. Mol Cell 2011; 42: 731-43.

25. Dong W, Xiao S, Cheng M, Ye X, Zheng G. Minocycline induces protective autophagy in vascular endothelial cells exposed to an in vitro model of ischemia/reperfusion-induced injury. Biomed Rep 2016; 4: 173-7.

26. Wang G, Liu K, Li Y, et al. Endoplasmic reticulum stress mediates the anti-inflammatory effect of ethyl pyruvate in endothelial cells. PLoS One 2014; 9: e113983.

27. Liu D, Xu L, Zhang X, et al. Snapshot: Implications for mTOR in aging-related ischemia/reperfusion injury. Aging Dis 2019; 10: 116-33.

28. Chauhan A, Sharma U, Jagannathan NR, Reeta KH, Gupta YK. Rapamycin protects against middle cerebral artery occlusion induced focal cerebral ischemia in rats. Behav Brain Res 2011; 225: 603-9. 
29. Meloni BP, Meade AJ, Kitikomolsuk D, Knuckey NW. Characterization of neuronal cell death in acute and delayed in vitro ischemia (oxygen-glucose deprivation) models. J Neurosci Methods 2011; 195: 67-74.

30. Han J, Pan XY, Xu Y, et al. Curcumin induces autophagy to protect vascular endothelial cell survival from oxidative stress damage. Autophagy 2012; 8: 812-25.

31. Li Z, Li J, Tang N. Long noncoding RNA Malat1 is a potent autophagy inducer protecting brain microvascular endothelial cells against oxygen-glucose deprivation/ reoxygenation-induced injury by sponging miR-26b and upregulating ULK2 expression. Neuroscience 2017; 354: $1-10$.

32. Chichger H, Rounds S, Harrington EO. Endosomes and autophagy: regulators of pulmonary endothelial cell homeostasis in health and disease. Antioxid Redox Signal 2019; 31: 994-1008.

33. Xie Y, You SJ, Zhang YL, et al. Protective role of autophagy in AGE-induced early injury of human vascular endothelial cells. Mol Med Rep 2011; 4: 459-64.

34. Into T, Horie T, Inomata M, et al. Basal autophagy prevents autoactivation or enhancement of inflammatory signals by targeting monomeric MyD88. Sci Rep 2017; 7: 1009.

35. Kim KA, Shin D, Kim JH, et al. Role of autophagy in endothelial damage and blood-brain barrier disruption in ischemic stroke. Stroke 2018; 49: 1571-9.

36. Xie R, Li X, Ling Y, et al. Alpha-lipoic acid pre- and post-treatments provide protection against in vitro ischemia-reperfusion injury in cerebral endothelial cells via Akt/mTOR signaling. Brain Res 2012; 1482: 81-90.

37. Wong PM, Puente C, Ganley IG, Jiang X. The ULK1 complex: sensing nutrient signals for autophagy activation. Autophagy 2013; 9: 124-37.

38. Carloni S, Buonocore G, Longini M, Proietti F, Balduini W. Inhibition of rapamycin-induced autophagy causes necrotic cell death associated with Bax/Bad mitochondrial translocation. Neuroscience 2012; 203: 160-9.

39. Carloni S, Albertini MC, Galluzzi L, Buonocore G, Proietti F, Balduini W. Increased autophagy reduces endoplasmic reticulum stress after neonatal hypoxia-ischemia: role of protein synthesis and autophagic pathways. Exp Neurol 2014; 255: 103-12.

40. Sheng R, Liu XQ, Zhang LS, et al. Autophagy regulates endoplasmic reticulum stress in ischemic preconditioning. Autophagy 2012; 8: 310-25.

41. Appenzeller-Herzog C, Hall MN. Bidirectional crosstalk between endoplasmic reticulum stress and mTOR signaling. Trends Cell Biol 2012; 22: 274-82.

42. Kato H, Nakajima S, Saito Y, Takahashi S, Katoh R, Kitamura M. mTORC1 serves ER stress-triggered apoptosis via selective activation of the IRE1-JNK pathway. Cell Death Differ 2012; 19: 310-20.

43. Ogata M, Hino S, Saito A, et al. Autophagy is activated for cell survival after endoplasmic reticulum stress. Mol Cell Biol 2006; 26: 9220-31.

44. Zhu J, Hua X, Li D, Zhang J, Xia Q. Rapamycin attenuates mouse liver ischemia and reperfusion injury by inhibiting endoplasmic reticulum stress. Transplant Proc 2015; 47: 1646-52.

45. Kapuy O, Vinod PK, Bánhegyi G. mTOR inhibition increases cell viability via autophagy induction during endoplasmic reticulum stress - an experimental and modeling study. FEBS Open Bio 2014; 4: 704-13.

46. Foster DA, Toschi A. Targeting mTOR with rapamycin: one dose does not fit all. Cell Cycle 2009; 8: 1026-9. 